\title{
Valoración de propiedades físicas del pet y maderas como alternativa para el uso en fortificación minera
}

\author{
Feijoo Calle Ernesto Patricio \\ ORCID: https://orcid.org/0000-0001-6901-7933 \\ pfeijoo@uazuay.edu.ec \\ Universidad del Azuay \\ Cuenca-Ecuador
}

\author{
Núñez Rodas Leonardo Aníbal \\ ORCID: https://orcid.org/0000-0003-4730-6114 \\ lnunez@uazuay.edu.ec \\ Universidad del Azuay \\ Cuenca-Ecuador
}

Recibido (04/02/21), Aceptado (23/02/21)

Resumen: El siguiente trabajo tiene como objetivo determinar si el polietileno de tereftalato (PET) reciclado, con la adición de agregados finos (arena), es una alternativa efectiva al uso de madera, en estructuras de fortificación en pequeña minería subterránea, evaluando las propiedades físicas de resistencia a la compresión, resistencia a la flexión, resistencia al pandeo y resistencia al corte. Para conseguir este propósito fue necesario el diseño y elaboración de probetas compuestas por el PET reciclado y una carga reforzante de arena utilizada para la construcción, así mismo se elaboraron probetas de las maderas más usadas para fortificación, eucalipto, teca y pino. Los datos y cálculos respectivos permitieron elaborar fichas técnicas en las que se describen la composición, características y observaciones que se recopilaron de cada muestra elaborada y tratada. Los resultados son concluyentes en las diferentes propiedades físicas y se hacen recomendaciones para una nueva etapa de investigación.

Palabras Clave: Fortificación, PET, minería, reciclaje.

\section{Assessment of the physical properties of pet and wood as an alternative for use in mining fortification}

\begin{abstract}
This work aims to determine if recycled polyethylene terephthalate (PET), with the addition of fine aggregates (sand), is an effective alternative to the use of wood, in fortification structures in small underground mining, evaluating the physical properties of compressive strength, flexural strength, buckling strength and shear strength. To achieve this purpose, it was necessary to design and manufacture test tubes made of recycled PET and a reinforcing load of sand used for construction, as well as test tubes of the most used woods for fortification, eucalyptus, teak and pine. The respective data and calculations allowed the elaboration of technical sheets in which the composition, characteristics and observations that were collected from each sample prepared and treated are described. The results are conclusive in the different physical properties and recommendations are made for a new stage of investigation.
\end{abstract}

Keywords: Fortification, PET, mining, recycling. 


\section{INTRODUCCIÓN}

La minería ha ocupado un lugar prioritario en el desarrollo y evolución de la vida humana, hasta llegar a lo que hoy conocemos como civilización, pero siempre ha involucrado el uso y posterior desecho de materiales, generando situaciones poco sostenibles y sustentables, específicamente en la minería subterránea, se ha optado por utilizar diferentes maderas, con el fin de fortificar los emplazamientos y túneles para estabilizarlos y evitar el desprendimiento de material rocoso para generar seguridad a personas y equipos. Es por esto, que la utilización de un material no natural y reciclado, que suplante a la madera, se piensa deseable y para lograr aquello se propone estudiar al polietileno de tereftalato (PET) usado en envases de alimentos o bebidas; este material totalmente reciclable ya se ha utilizado en otro tipo de productos sustentables [1] y en esta investigación se intenta probar que puede sustituir a las maderas. Para esto se han elaborado una serie de probetas compuestas por PET con una variación en porcentaje y tamaño de granos de arena, dichas probetas serán sometidas a pruebas y ensayos pertinentes, a fin de probar si existe viabilidad técnica para la suplantación de pilares de madera por pilares de PET reciclado y evitar el uso de un recurso natural. Los resultados serán presentados en gráficas que detallen su comportamiento y caracterización a fin de identificar el prototipo adecuado que pueda cumplir estas expectativas. De igual manera se elaboraron una serie de probetas de las maderas utilizadas en nuestro medio en la minería subterránea, las cuales son de las especies eucalipto, teca y pino. Dada la importancia de la recopilación bibliográfica para entender diferentes temas que se subdividen en la investigación acerca de las variables que la componen, se puede afirmar que el comportamiento de los plásticos, su codificación y origen son temas de vital importancia [2], pasando a través de sus clasificaciones y características más notables, así como los elementos que pueden complementarlo y crearlo, este es el caso de las cargas reforzantes en la investigación, todo esto se indaga con el fin de tener una base sustentable de información sobre propiedades específicas que se utilizarán en los métodos de experimentación.

\section{II.DESARROLLO}

Iniciamos con una descripción de los materiales y la metodología usada.

El polietileno de tereftalato (PET), figura 1, posee características físicas y propiedades mecánicas muy buenas, como su dureza, su peso ligero, su resistencia al desgaste, su fácil moldeo y adaptación a diferentes aplicaciones [3]. Para el diseño de las mezclas se tomó en consideración que la composición de las probetas debía contener un elevado porcentaje de PET, debido a que su uso como material principal era un motivo para incentivar el reciclaje y dar un nuevo uso a uno de los polímeros más producidos, usados y desechados de la industria.

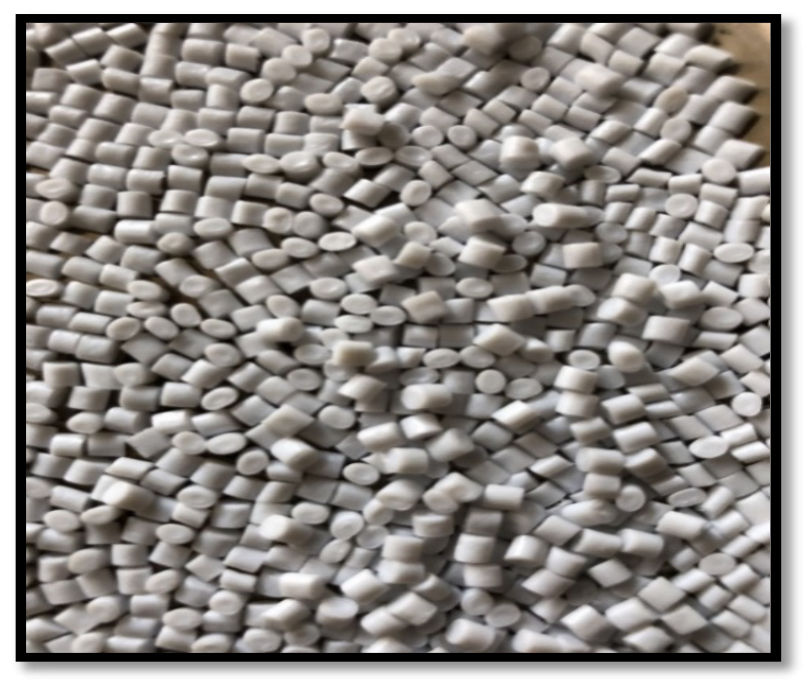

Fig. 1. PET en gránulos.

La arena usada en la mezcla y elaboración de las probetas proviene de un sector denominado El Tahual, en la provincia del Azuay (Ecuador), específicamente es extraída de la formación Biblián, sus afloramientos se dan en los núcleos del anticlinal de Cuenca, sinclinal de Azogues y en el margen centro sureste de la cuenca. La litología del terreno se puede definir como una secuencia sedimentaria clástica que presenta alternancia de argilitas, limolitas rojizas y areniscas tobáceas de grano fino a grueso, también se presentan conglomerados de cantos mal sorteados y subangulares de la formación Yunguilla [4]. (Figura 2). 


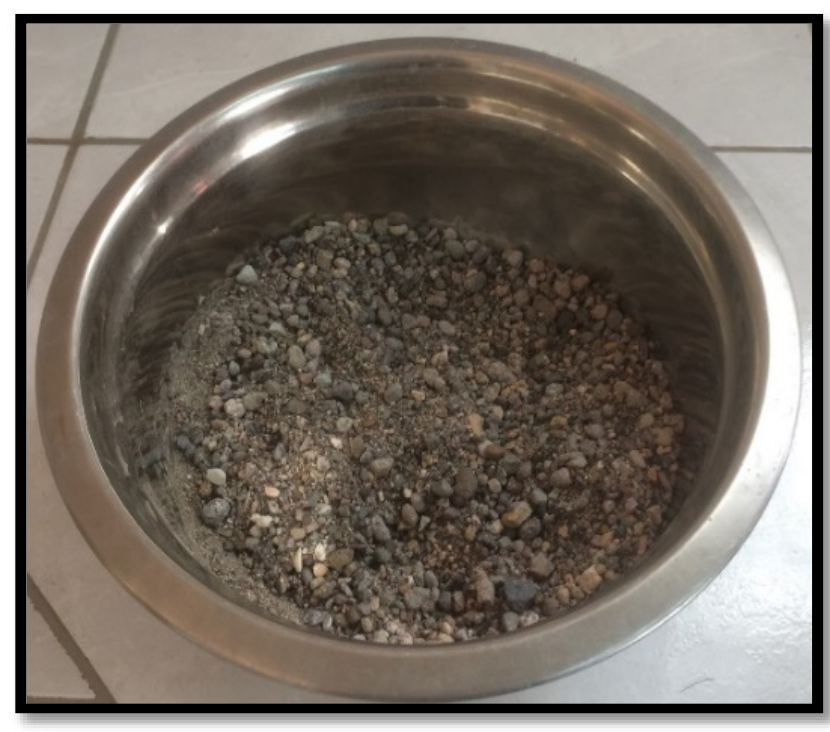

Fig. 2. Arena para la mezcla.

El eucalipto es una especie de la Familia Myrtaceae, de tronco recto y cilíndrico, de color amarillo pálido y vetado poco diferenciado, alcanzan alturas entre los 45 y $70 \mathrm{~m}$. La durabilidad del duramen es superior al de la albura, además es adecuado para el cepillado, taladrado y enclavado. Para fuerzas de flexión, compresión y tracción es muy resistente, pero necesita cuidado en el pulido. Durante los 2 primeros años crece en diámetro como en altura, después de eso solo la copa crece en diámetro. Su tiempo de crecimiento es entre 13 a 15 años. Es resistente al fuego [5]. (Figura 3).

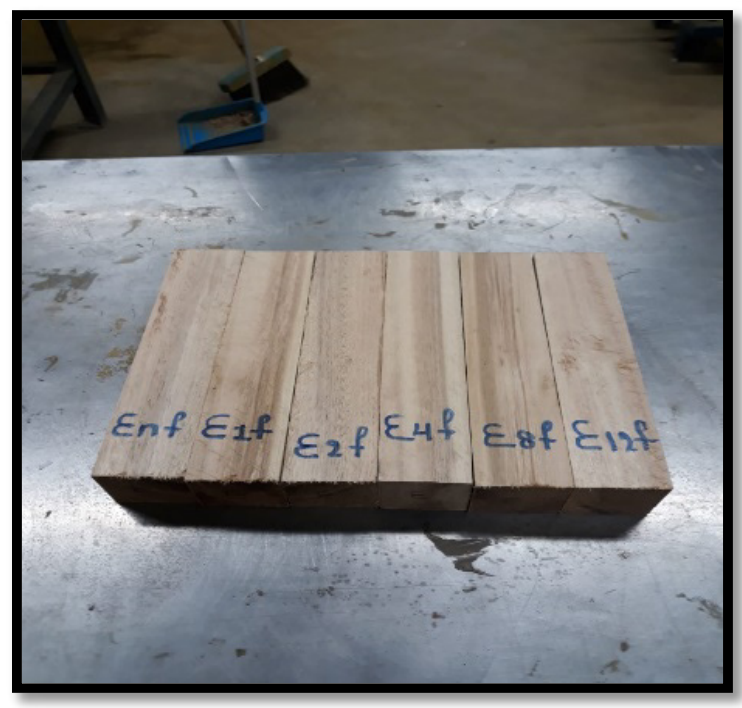

Fig. 3. Probetas de eucalipto.

La madera de teca es muy duradera y resistente, debido al aceite que contiene que la hace muy impermea- ble y la protege de los ataques de insectos y hongos. Todo ello hace que sea muy utilizada en exteriores, en estructuras para la construcción de viviendas y otros usos. Toda la madera de Teca proviene de plantaciones, lo que provoca un amplio beneficio ambiental y social al generar empleos directos e indirectos y restablecer ambientes degradados por la deforestación de la vegetación natural [6]. Figura 4.

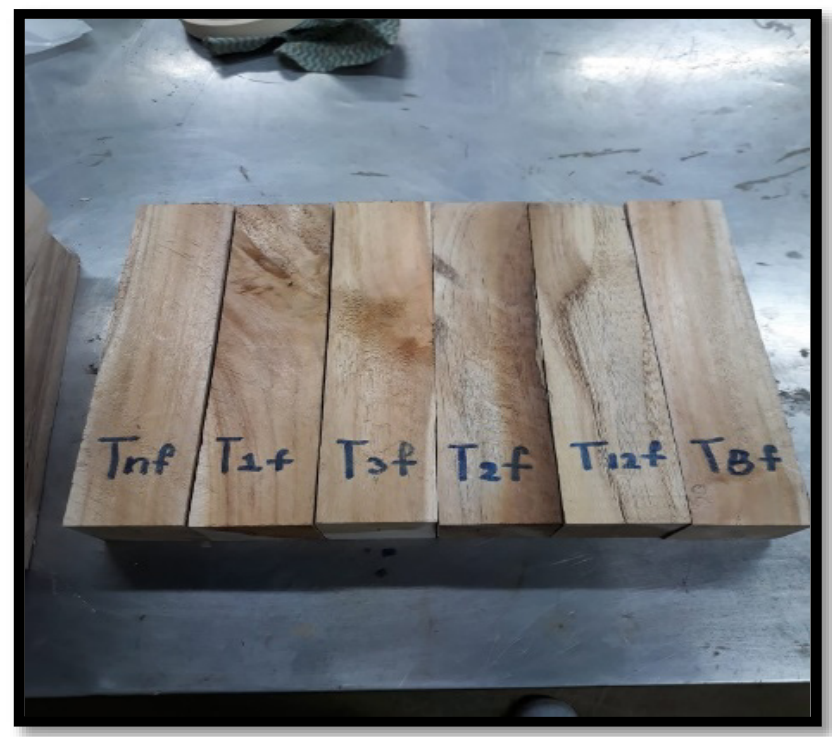

Fig. 4. Probetas de teca.

El pino pertenece a la Familia Pinaceae, su tronco es recto y cilíndrico, llegan a medir entre 20 a 40 metros de altura, mientras que su diámetro va de 40 a $150 \mathrm{~cm}$, su crecimiento en altura culmina a los 25 años, aproximadamente. Es susceptible al ataque de hongos y xilófagos [7]. (Figura 5).

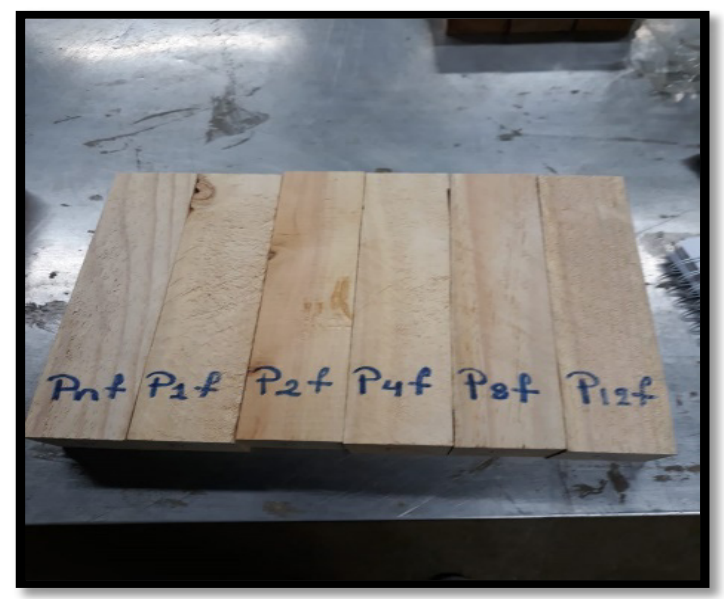

Fig. 5. Probetas de pino. 
En una segunda fase se realizó la preparación de los materiales y elaboración de las probetas.

Las probetas se elaboraron con variables en composición y en tamaño del agregado, con las siguientes dimensiones: $25 \mathrm{~cm} \times 5 \mathrm{~cm} \times 5 \mathrm{~cm}$, con las variaciones de 70,80 y $90 \%$ de PET y granulometrías para la arena con tamiz 30, tamiz 40 y tamiz 50. En la tabla I se pueden observar las diferentes probetas con sus composiciones. Cabe indicar que de estas probetas se elaboraron 4 conjuntos para los cuatro diferentes tipos de ensayos.

Tabla I. Composición de las probetas de PET y agregados

\begin{tabular}{|c|c|c|c|}
\hline Probeta & PET (\%) & Arena (\%) & Tamiz (\#) \\
\hline PET $10 / 50$ & 90 & 10 & 50 \\
\hline PET 20/50 & 80 & 20 & 50 \\
\hline PET 30/50 & 70 & 30 & 50 \\
\hline PET 10/40 & 90 & 10 & 40 \\
\hline PET 20/40 & 80 & 20 & 40 \\
\hline PET 30/40 & 70 & 30 & 40 \\
\hline PET 10/30 & 90 & 10 & 30 \\
\hline PET 20/30 & 80 & 20 & 30 \\
\hline PET 30/30 & 70 & 30 & 30 \\
\hline
\end{tabular}

Por ejemplo, la probeta PET 30/50 nos indica que la misma contiene el $70 \%$ de PET y el $30 \%$ de arena pasante el tamiz número 50. A más de las 9 muestras descritas se elaboró una muestra con el $100 \%$ de PET, sin ningún tipo de mezcla. Estas probetas se las puede observar en la figura 6. Con las mismas dimensiones se elaboraron las probetas de eucalipto, teca y pino del mismo árbol y siguiendo la misma dirección y diámetro de cada tronco, para lograr tener probetas de similares características.

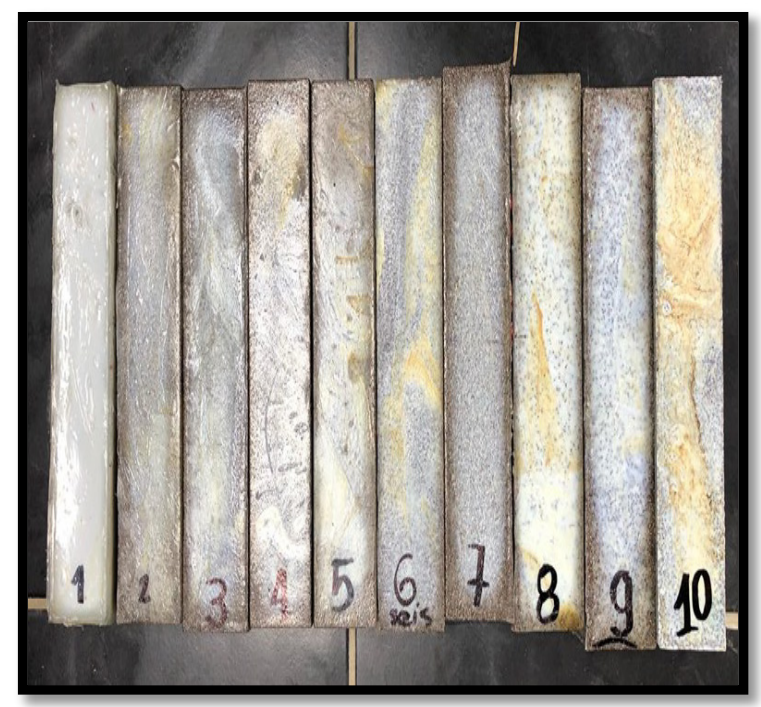

Fig. 6. Probetas de PET y agregado.

El procedimiento de preparación de la arena se inició con un análisis granulométrico de las muestras con un peso aproximado de 2.5 kilogramos cada una. Después del análisis granulométrico respectivo, se realizaron 
procesos tales como una preparación física, lavado, secado, tamizado, pesaje y realización de cálculos; se procedió a determinar cuáles son los tamaños de partícula o granulometría que serán utilizadas como agregado en la elaboración de las probetas.

El PET se obtiene a través de diferentes procesos de extrusión, inyección y soplado, este material polimérico al ser higroscópico puede absorber la humedad del ambiente por lo cual es introducido en un secador térmico que eleva la temperatura por encima de $\operatorname{los} 100^{\circ} \mathrm{C}$, a fin de evaporar cualquier humedad que pueda generar porosidad o aglutinamiento en el momento de la fundición y polimerización del polímero con el agregado.

Al tener la arena y el PET, con las características físicas que se requieren, se procede a encender dos hornos, el primero a una temperatura de $350 \mathrm{oC}$ y el segundo a una temperatura de $50 \mathrm{oC}$.

En un recipiente apto para el calor se coloca el porcentaje de arena de cada muestra, y se la ingresa en el horno a $350 \mathrm{oC}$, por aproximadamente 3 minutos. A la arena caliente se le agrega el porcentaje correspondiente a cada muestra de PET y se la coloca en el horno a 350 oC, se deja esta mezcla cinco minutos en el horno, para que el PET se comience a derretir, se saca el recipiente del horno y se comienza a homogenizar la mezcla mediante movimientos circulares con una varilla de hierro resistente al calor para luego volver a introducir el recipiente en el horno.

Se realiza este procedimiento entre cuatro a cinco veces con el mismo intervalo de tiempo, cinco minutos, para obtener una mezcla totalmente homogénea y fluida.

Al tener esta mezcla se la coloca en un molde de acero de transmisión para altas temperaturas, cuyas medidas son de $25 \mathrm{~cm}$ de longitud x $5 \mathrm{~cm}$ de ancho $\mathrm{x} 5 \mathrm{~cm}$ de profundidad, para que la mezcla tome la forma del molde. Este molde debe estar humedecido con aceite y tener la misma temperatura que la muestra, para que no se solidifique antes de proporcionarle la presión necesaria, al momento de apretar los tornillos. Finalmente, el molde se deja a temperatura ambiente por cinco minutos, luego se procede a desarmar los tornillos y retirar la muestra, para de manera inmediata, introducirla en el horno a $50 \mathrm{oC}$, y así obtener un proceso de enfriamiento controlado, esto debido a que, si la muestra se enfría abruptamente, se cuartea y/o rompe, volviéndola inservible.

Cabe recalcar que paralelamente se elaboraron las probetas de las diferentes maderas.

Finalmente se ejecutaron los distintos ensayos.

La resistencia a la compresión simple es el esfuerzo máximo que puede soportar un material a una fuerza de compresión. Dependiendo del material que compongan las muestras se puede decir que este ensayo proporciona la cantidad de esfuerzo necesario para deformar o romper un material y se calcula al dividir la carga máxima que soporta para el área transversal de la muestra cilíndrica. Para ejecutar el ensayo de compresión simple es necesario la utilización de una prensa hidráulica Humboldt con capacidad de ejercer una carga de aplastamiento de hasta 50000 kilogramos de fuerza.

La flexión es una combinación de esfuerzos de compresión y de tracción. Mientras que las fibras superiores de la probeta están sometidas a un esfuerzo de flexión (se alargan), las inferiores se acortan, o viceversa, produciendo una deformación a lo largo de su eje, que tiende a doblarlo. El rasgo más destacado es que un objeto sometido a flexión presenta una superficie de puntos llamada fibra neutra tal que la distancia a lo largo de cualquier curva contenida en ella, no varía con respecto al valor antes de la deformación.

El pandeo se produce cuando superan la resistencia las piezas sometidas al esfuerzo de compresión en el sentido de sus fibras, generando una fuerza perpendicular a ésta, produciendo que se doble en la zona de menor resistencia.

La aparición de dicha flexión lateral, su rápido crecimiento y la pérdida total de estabilidad del elemento y el consiguiente colapso de la estructura, constituyen el análisis al pandeo.

Cuando se aplican fuerzas perpendiculares al eje del elemento, existe la tendencia de cortado a través de su sección transversal, produciendo un esfuerzo cortante. Si una sola sección transversal del elemento resiste la fuerza aplicada, se dice que se produce un efecto de cortante simple.

\section{III.RESULTADOS}

Luego de ejecutados todos los ensayos de laboratorio y realizados los respectivos cálculos, se presentan los resultados en la Tabla II y en las figuras 7, 8, 9 y 10, haciendo una comparación para las diferentes propiedades [8]-[9]-[10]-[11]-[12]-[13]-[14].

En la Tabla II se recopilan todos los resultados de los diferentes ensayos con sus respectivas denominaciones de las probetas.

En las figuras 7, 8, 9 y 10 se presenta una comparación de los valores de compresión, tensión, pandeo y corte, respectivamente de cada una de las probetas tratadas en cada ensayo y podemos observar cual probeta fue la que presentó más resistencia en cada propiedad, este valor mayor se lo ha representado en color verde para diferenciarlo, cabe indicar que todos los valores de las propiedades descritas están en mega pascales. 
Se observa que solamente en la prueba de resistencia a la compresión las probetas de PET logran tener valores mayores que las probetas de maderas, el resto de propiedades son superadas notablemente por las probetas de madera, lo cual genera preguntas para realizar propuestas de mejoramiento al respecto.

Tabla 2. Resultados de los ensayos

\begin{tabular}{|c|c|c|c|c|}
\hline $\begin{array}{c}\text { Ensayo } \\
\text { Probeta }\end{array}$ & $\begin{array}{c}\text { Compresión } \\
\mathrm{MPa}\end{array}$ & $\begin{array}{c}\text { Flexión } \\
\mathrm{MPa}\end{array}$ & $\begin{array}{c}\text { Pandeo } \\
\mathrm{MPa}\end{array}$ & $\begin{array}{c}\text { Corte } \\
\mathrm{MPa}\end{array}$ \\
\hline PET & 75.80 & 13.20 & 55.60 & 5.99 \\
\hline PET 10/50 & 75.30 & 19.50 & 27.40 & 2.28 \\
\hline PET 10/40 & 65.90 & 25.50 & 64.10 & 6.63 \\
\hline PET 10/30 & 47.50 & 28.80 & 70.20 & 5.64 \\
\hline PET 20/50 & 69.40 & 16.10 & 32.40 & 5.25 \\
\hline PET 20/40 & 35.90 & 32.60 & 74.80 & 3.48 \\
\hline PET 20/30 & 65.80 & 16.00 & 62.60 & 4.28 \\
\hline PET 30/50 & 64.30 & 15.60 & 26.30 & 4.33 \\
\hline PET 30/40 & 59.00 & 16.30 & 38.20 & 5.76 \\
\hline PET 30/30 & 53.90 & 8.20 & 54.00 & 5.02 \\
\hline EUCALIPTO & 60.10 & 105.8 & 79.80 & 17.78 \\
\hline TECA & 51.40 & 55.40 & 54.60 & 11.73 \\
\hline PINO & 21.70 & 33.10 & 37.01 & 9.35 \\
\hline
\end{tabular}

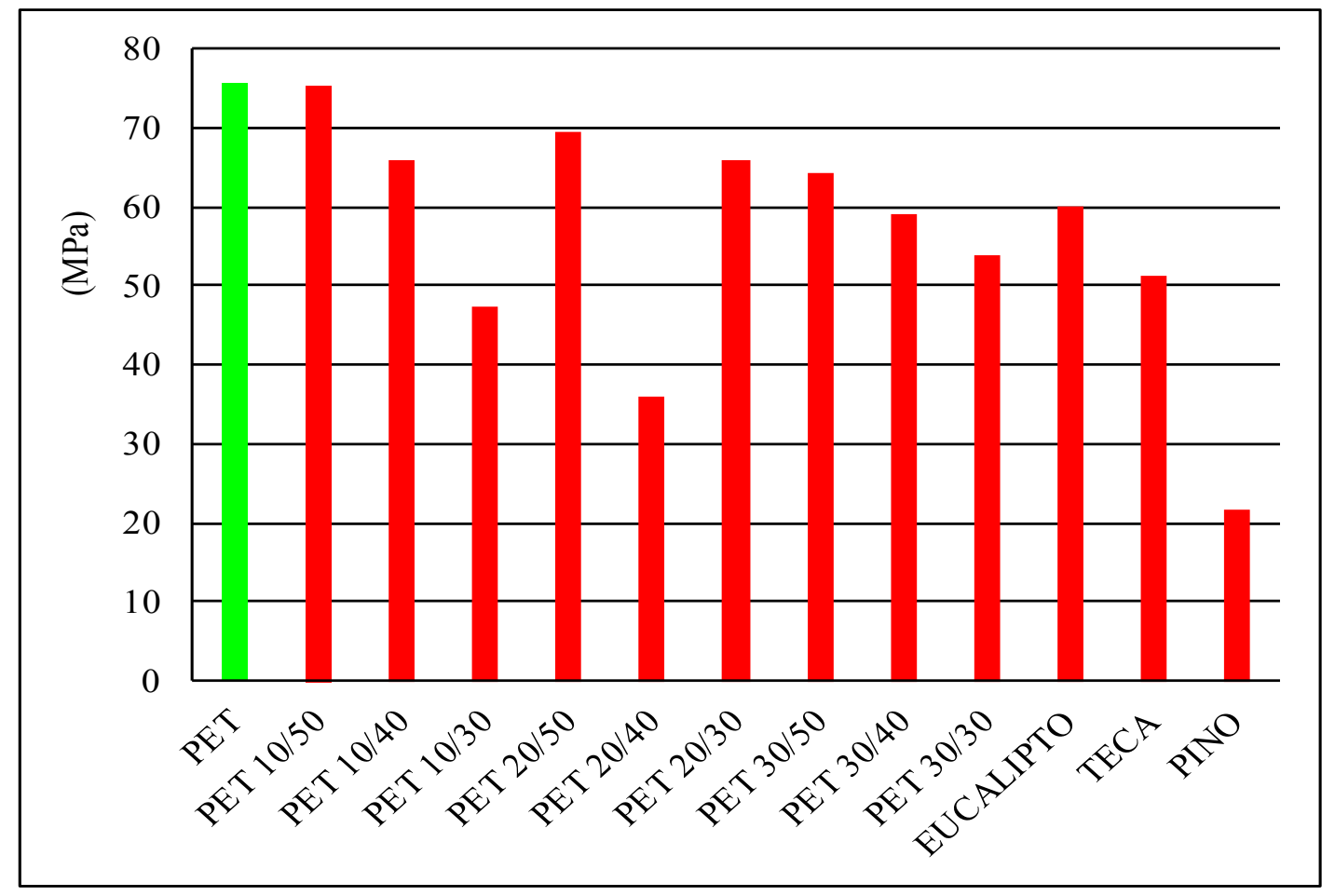

Fig. 7. Datos de valores de resistencia a la compresión. 


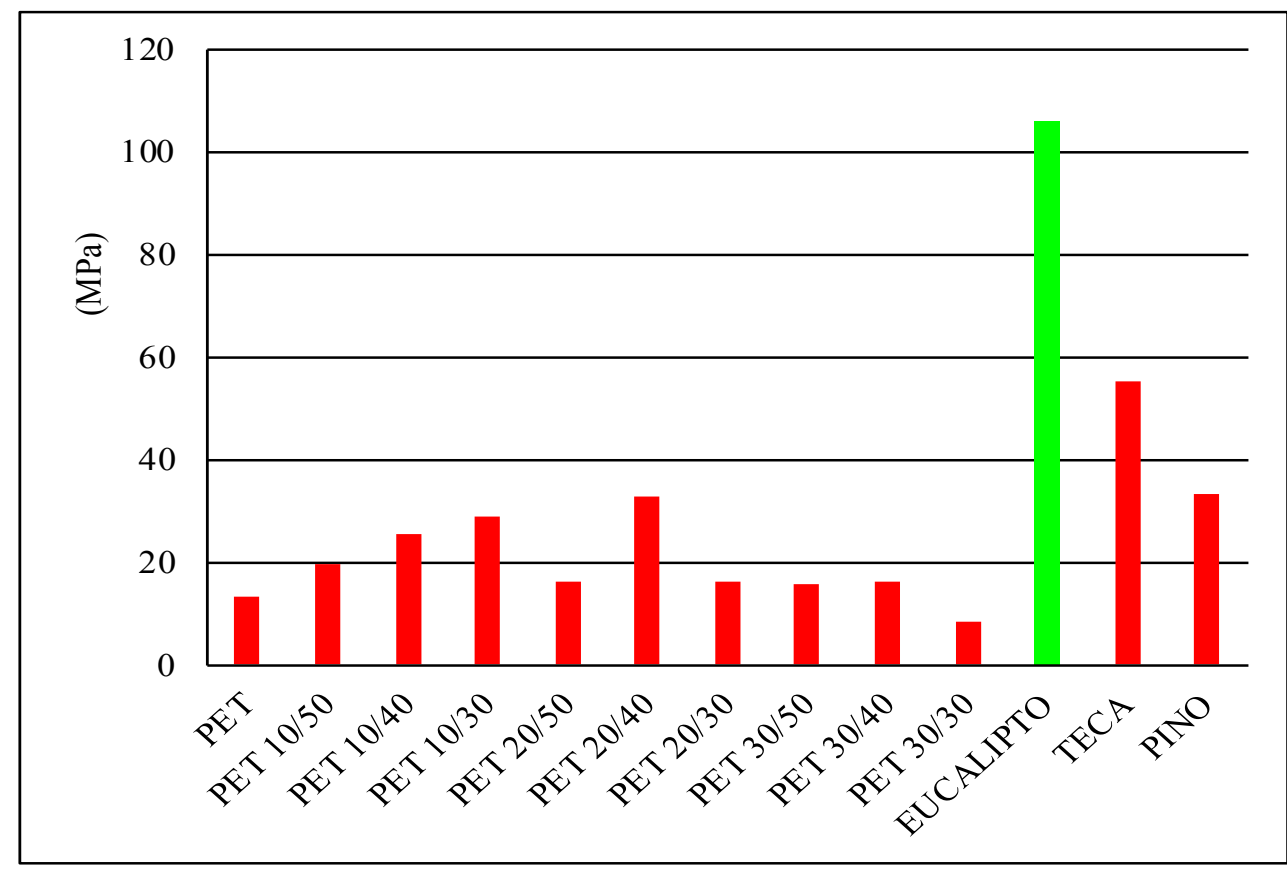

Fig. 8. Datos de valores de resistencia a la flexión.

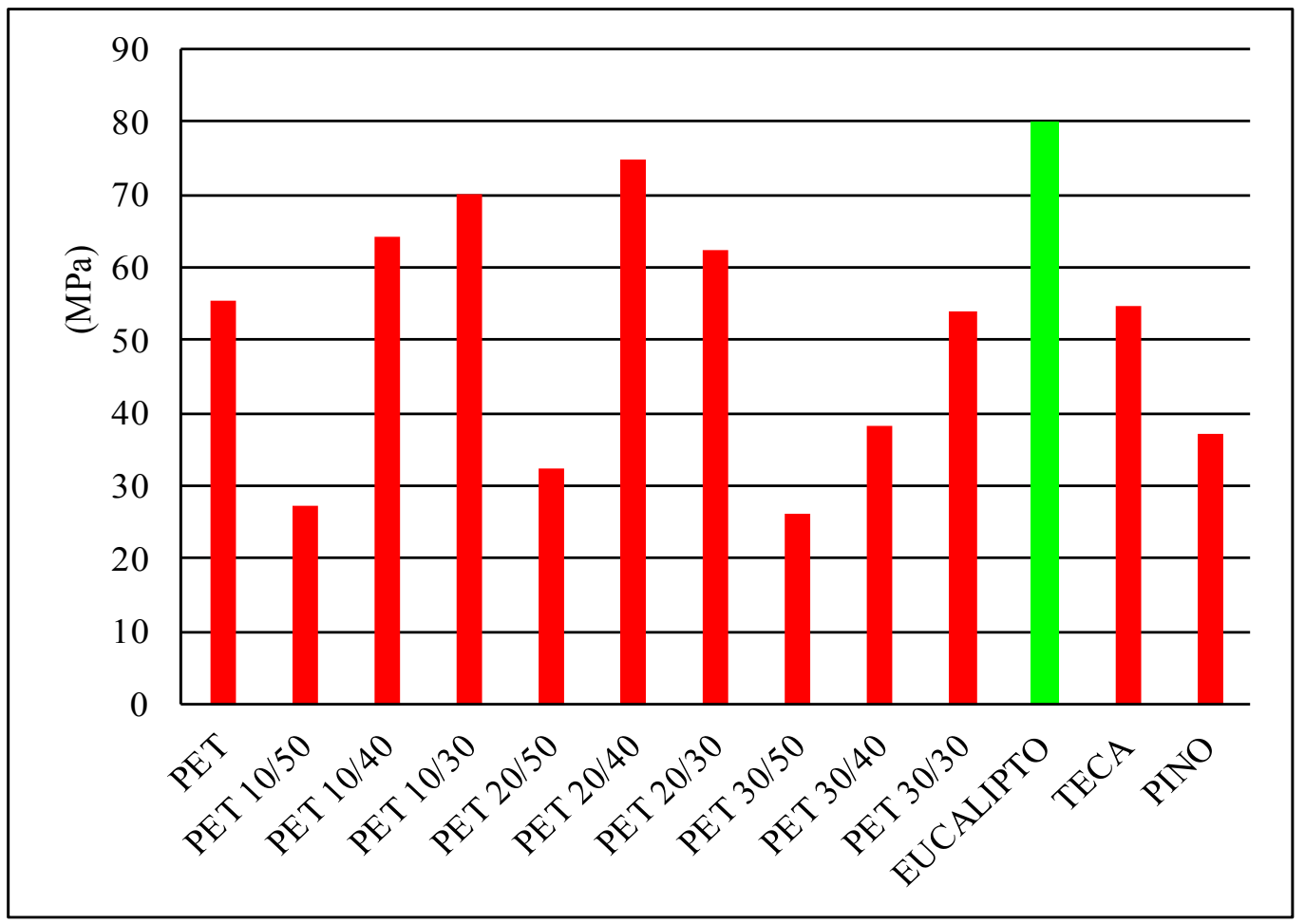

Fig. 9. Datos de valores de resistencia al pandeo. 


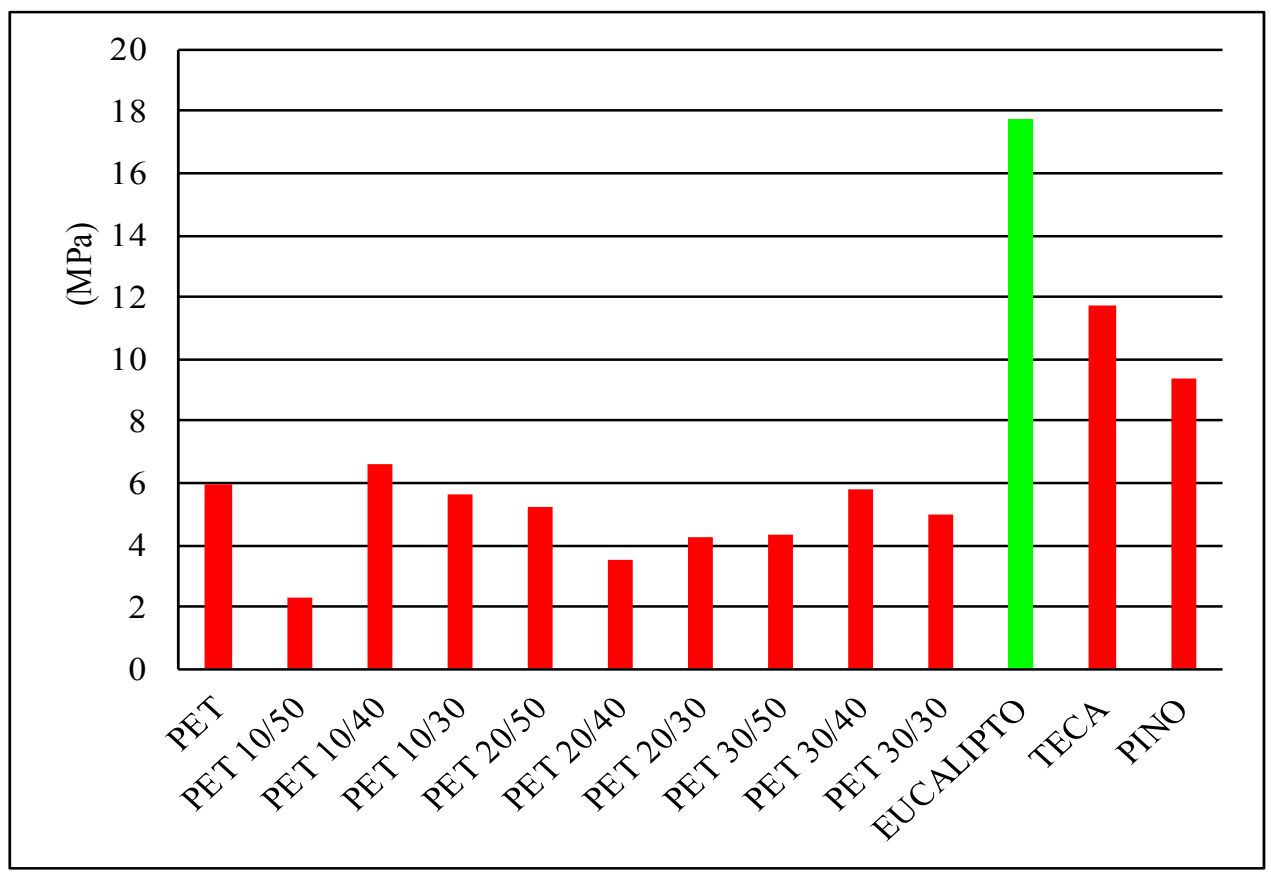

Fig. 10. Datos de valores de resistencia al corte.

\section{III.CONCLUSIONES}

En la figura 7 se puede observar que la probeta de PET al $100 \%$ es la que mayor resistencia a la compresión obtuvo en el ensayo respectivo, sin embargo, la probeta PET 10/50 alcanzó similar resistencia, por lo que estaría produciéndose un buen elemento para un estudio más detallado al respecto y poder ser el sustituto propuesto, con la finalidad de evitar un consumo excesivo de madera en el distrito minero.

Se puede observar en la figura 9 que las probetas de PET y agregado no logran valores similares a los de las maderas en el ensayo de pandeo, pero la probeta PET 20/40 está cerca de ese objetivo, por lo que se podría pensar en un elemento de sustitución de la madera.

Las probetas de PET y agregados, presentan resistencias muy bajas en las pruebas de flexión y corte, figuras 8 y 10, por lo que para mejorar estos ensayos se debería agregar otro material a la mezcla y de esta forma suplir esta deficiencia.

El eucalipto es la madera que presentó mayores valores en las pruebas de flexión, pandeo y corte, figuras 8,9 y 10 , a más de que presenta una buena resistencia a la compresión; esto hace razonable que sea la madera más usada en nuestro medio para fortificación en pequeña minería.

\section{REFERENCIAS}

[1]B. Gnauk, C. Frundt, Iniciación a la Química de los
Plásticos, Barcelona, Hanser, 1989.

[2]R. Modernos, Ciencia y Tecnología de los Materiales Plásticos. Madrid, CSIC, 1990.

[3]R. Gómez, J. Gil Bercero, Los Plásticos y el Tratamiento de sus Residuos. Universidad Nacional de Educación a Distancia, 1997.

[4]M. Casanova, W. Vera, W. Leighton, O. Salazar, Guía de Edafología. Santiago, Universidad de Chile, 2004.

[5]I. Vinueza, Ecuador Forestal, http://ecuadorforestal. org/fichas-tecnicas-de-especies-forestales/ficha-tecnica-no-15-eucalyptus-globulus-labill/, 2003.

[6]I. Vinueza, Ecuador Forestal, http://ecuadorforestal. org/fichas-tecnicas-de-especies-forestales/ficha-tecnica-no-6-guayacan, 2012.

[7]E. Criollo, Mejoramiento de la calidad de la madera a utilizar en fortificaciones interior mina, Tesis de Grado, Universidad del Azuay, Ecuador, 2017.

[8]D. Rodríguez, Determinación del Uso de Plástico PET como Alternativa al uso de Madera en Fortificación Minera, Tesis de Grado, Universidad del Azuay, Ecuador, 2018.

[9]A. Rivera, Alternativas del uso de maderas en fortificación minera bajo diferentes condiciones de humedad, Tesis de Grado, Universidad del Azuay, Ecuador, 2018. [10]A. Botina, Alternativa de uso del PET con agregados (arena) en Fortificación Minera, mediante la caracterización de Resistencia a la Flexión, Tesis de Grado, Universidad del Azuay, Ecuador, 2019. 
[11]D. Nugra, Alternativas del uso de maderas en fortificación minera, mediante la caracterización de Resistencia a la Flexión, Tesis de Grado, Universidad del Azuay, Ecuador, 2019.

[12]K. Malla, Alternativa del uso del Polietileno de Tereftalato (PET) y agregados en Fortificación Minera en función de la Resistencia al Pandeo, Tesis de Grado, Universidad del Azuay, Ecuador, 2019.

[13]S. Hidalgo, G. Chávez, Comparación de la Resistencia al Esfuerzo Cortante entre el PET y Maderas utilizadas en Fortificación Minera, Tesis de Grado, Universidad del Azuay, Ecuador, 2019.

[14]K. Enderica, Caracterización de la Resistencia al Pandeo de las maderas más utilizadas en fortificación de túneles, bajo diferentes condiciones de humedad, Tesis de Grado, Universidad del Azuay, Ecuador, 2019.

\section{RESUMEN CURRICULAR}

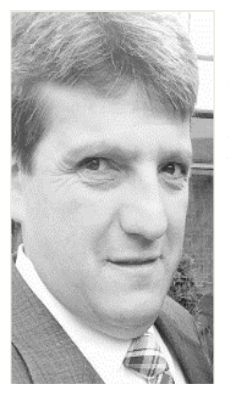

Patricio Feijoo C. Ingeniero en Minas, graduado en la Universidad del Azuay (Cuenca -Ecuador), con estudios y pasantías en: Bolivia, Brasil, España, Australia en áreas de la geología, geofísica y desarrollo de actividades de explotación de minas. Desde el año 1991 se encuentra vinculado a la docencia en la Universidad del Azuay, mantiene el cargo de Profesor Titular e Investigador.

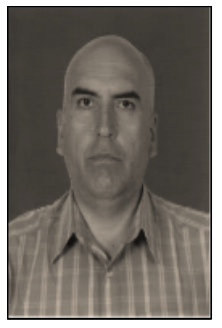

Leonardo Núñez R. Ingeniero en Minas, graduado en la Universidad Central del Ecuador (Quito - Ecuador), con estudios y pasantías en: Ecuador, Chile, Bolivia, Brasil y Colombia en las áreas de la investigación geológica, explotación minera, procesos metalúrgicos y gestión ambiental. Se encuentra vinculado a la docencia en la Universidad del Azuay, mantiene actualmente el cargo de Profesor Titular e Investigador. 